\title{
Feasibility and reliability of electrical, mechanical and thermal nociceptive testing and assessment of diffuse noxious inhibitory control in dogs
}

This article was published in the following Dove Press journal: Journal of Pain Research

\author{
Hélène LM Ruel ${ }^{1,2}$ \\ Ryota Watanabe ${ }^{1,2}$ \\ Marina C Evangelista ${ }^{1,2}$ \\ Guy Beauchamp ${ }^{3}$ \\ Paulo V Steagall ${ }^{1,2}$ \\ 'Department of Clinical Sciences, \\ Faculty of Veterinary Medicine, \\ Université de Montréal, Saint- \\ Hyacinthe, QC, Canada; ${ }^{2}$ Quebec \\ Animal Pharmacology Research \\ Group, Faculty of Veterinary Medicine, \\ Université de Montréal, Saint- \\ Hyacinthe, QC, Canada; ${ }^{3}$ Faculty \\ of Veterinary Medicine, Université \\ de Montréal, Saint-Hyacinthe, QC, \\ Canada
}

Purpose: Quantitative sensory testing has been used to assess the somatosensory system. This study aimed to evaluate the feasibility and reliability of electrical (ENT), mechanical (MNT) and thermal (TNT) nociceptive testing and the effect of a conditioning stimulus on MNT.

Patients and methods: Sixteen healthy client-owned dogs were included in this study. Stimulation was applied bilaterally to the dorsal and plantar aspect of the metacarpus and metatarsus respectively, using transcutaneous electrical stimulator, algometry and a cold nociceptive device in a randomized order until a behavior response was observed or a cut-off reached. Tests were performed twice ( 60 seconds apart) by two observers. Retesting was performed 5 hours later. The diffuse noxious inhibitory control was tested by comparing MNT pre- and post-conditioning stimuli. Sham-testing was performed for ENT and TNT. Statistical analysis included linear model and intra-class correlation coefficient $(P<0.05)$.

Results: Feasibility was 99\% (ENT), 93.5\% (MNT) and 93.6\% (TNT). Data for TNT were not analyzed due to inconsistent results. Mean \pm SD were $48 \pm 22.6 \mathrm{~mA}(\mathrm{ENT})$ and $11.9 \pm 3.5 \mathrm{~N}$ (MNT). MNT was higher for thoracic than for pelvic limbs $(P=0.002)$. Conditioning stimulus increased MNT $(P=0.049)$. Inter-observer reliability was $91.4 \%(\mathrm{ENT})$ and $60.9 \%(\mathrm{MNT})$. False-positive responses were 15\% (ENT) and 35.7\% (TNT).

Conclusion: ENT was feasible, repeatable and superior to MNT and TNT. The assessment of the diffuse noxious inhibitory control with a conditioning stimulus showed promising results in dogs. These tools could be used in naturally-occurring disease to provide insight on their underlying mechanisms and therapeutics.

Keywords: electrical threshold, mechanical threshold, thermal threshold, quantitative sensory testing, diffuse noxious inhibitory controls, canine

\section{Introduction}

Cutaneous sensations such as pain, temperature and touch are conveyed by three ascending pathways that are part of the somatosensory system in dogs, namely the spinothalamic, the spinocervicothalamic and the dorsal column postsynaptic tracts. ${ }^{1}$ The somatosensory system is involved in the transmission of sensory stimuli from peripheral receptors to the cerebral cortex. Quantitative sensory testing (QST) has been used to characterize sensory nerve dysfunction and to assess the somatosensory system. ${ }^{2}$ These tests use different modalities (thermal [TNT], mechanical [MNT] or electrical [ENT]) to determine nociceptive thresholds.

QST has been used to establish sensory phenotypes in people experiencing pain. ${ }^{3}$ These results have been used to predict response to therapy as part of personalized or precision medicine. For this reason, QST has gained interest in veterinary medicine and
Correspondence: Hélène LM Ruel Department of Clinical Sciences, Faculty of Veterinary Medicine, Université de Montréal, 3200 rue Sicotte, SaintHyacinthe, QC J2S 2M2, Canada Tel +I 4507788 III ext86550 Email helene.ruel.2@umontreal.ca 
it has been studied and validated in dogs and cats with osteoarthritis $^{4,5}$ and in dogs with thoracolumbar disc herniation. ${ }^{6}$ These studies concluded that QST is useful and feasible ${ }^{5-8}$ however, there is a lack of strong inter- and intra-observer reliability. Test-retest reliability has been evaluated in dogs with osteoarthritis and showed great individual variability over time. ${ }^{9}$ In addition, sham-testing is important to exclude potential false-positive responses in any nociceptive threshold testing. To the authors' knowledge, the latter has not been described for different QST modalities in dogs.

The aim of this study was to evaluate the feasibility and repeatability of electrical, thermal (cold) and mechanical nociceptive stimulation in healthy dogs, including shamtesting, for the first two modalities. In addition, the ability of a conditioning stimulus to assess the diffuse noxious inhibitory control (DNIC) using MNTs was tested. The hypothesis of this study was that electrical, thermal and mechanical nociceptive stimulation would be feasible with good intra- and inter-reliability in healthy dogs. The authors speculated that the conditioning stimulus would significantly increase MNT.

\section{Materials and methods}

This study was approved by the Animal Care Committee of the Faculty of Veterinary Medicine, Université de Montréal (16-Rech-1835), according to the Canadian Council of Animal Care guidelines. The study was performed at the Centre Hospitalier Universitaire Vétérinaire between October and December 2016.

\section{Animals}

Sixteen healthy client-owned dogs were recruited in a prospective, blinded, randomized clinical trial after obtaining owner's written consent. Patients were included if they were considered healthy based on physical, orthopedic and neurological examinations performed by the same observer (HLMR). Inclusion criteria included age (1-9 years old) and body weight ( $\geq 5 \mathrm{~kg}$ ). Exclusion criteria included pregnancy, lactation, aggression, anxiety, systemic disease (including obesity; body condition score $>7 / 9$ ), previous history of cancer/tumor removal, pacemaker placement, signs of pain or administration of analgesics 30 days prior to recruitment. Owners were asked to fill out a questionnaire to rule out issues with quality of life and chronic pain (Canine Brief Pain Inventory [CBPI] $).{ }^{10}$ Additionally, two observers assessed each dog separately and filled out the short-form Glasgow Composite Pain Measure Scale (CPMS-SF) ${ }^{11}$ before the experiments had begun. A total CPMS-SF score above the analgesic intervention score $(6 / 24)^{11}$ was considered as an exclusion criterion.
Dogs were admitted in the morning before the experiments and housed individually in kennels. Bedding and water ad libitum were provided.

\section{Observers}

Two veterinarians (observers; $\mathrm{Obs}_{1}$ and $\mathrm{Obs}_{2}$ ) were involved with QST in this study. These observers were responsible for identifying the behavioral response to nociceptive stimuli and for the application of mechanical nociceptive stimulation. They were not aware of stimuli intensity during testing. A third veterinarian who was not blinded to stimuli intensity was responsible for controlling thermal and electrical nociceptive stimulation (including sham-testing), randomization and compilation of results. This individual was also responsible for terminating the stimuli once cut-off was reached, or when a behavioral nociceptive response was identified by the first two observers. Both observers were unaware of randomization for sham-testing.

\section{Quantitative sensory testing}

QST was performed in the morning (first period) and repeated 5 hours later (second period) to evaluate intra- and inter-observer reliability of each nociceptive testing. Dogs were acclimated to the testing room for 10 minutes prior to the experimentation and had free access to water. Stimuli were applied on the dorsal aspect of the metacarpus and the plantar aspect of the metatarsus above the plantar pad bilaterally after clipping. These sites correspond to consistent autonomous zone of the radial and tibial nerve, respectively. ${ }^{12}$ Each observer repeated each QST modality twice (replicates) within the same period ( 60 seconds apart) for all limbs. The sequence of nociceptive testing, the order of the observers and the side (left or right) to be stimulated were randomized according to a random permutation generator (www.randomization.com). The latter was also used to randomly allocate the sham over the two periods (first or second period) of measurements for each observer. Dogs were kept in either a semi-sternal position or lateral recumbency over a mat during experiments to minimize handling.

Endpoints during nociceptive stimulation were characterized by behavioral responses including voluntary movement of the limb away from the probe, looking at the probe, vocalization or when a cut-off was reached. ${ }^{7}$ Rhythmic muscle contractions, withdrawal reflexes, or movement of a nonstimulated limb were not considered as positive responses. The following modalities of QST were used in this study:

a. Transcutaneous electrical nerve stimulator (TENS) for testing ENT - two self-adhesive electrodes (Dura-stick $®$ 
round 1.25", Chattanooga, Guildford, Surrey, UK) using a thin layer of conductivity gel were placed on the skin at the site of stimulation and secured in place with medical tape. The mode (VMSTM) was selected and delivered a symmetrical biphasic waveform with a $100 \mu \mathrm{sec}$ interphase. Settings were then adjusted to a $\mathrm{CC}$ mode using a frequency of $200 \mathrm{~Hz}$, phase duration $20 \mu \mathrm{sec}$ and ramp 0 seconds (Intelect ${ }^{\circledR}$ Vet two channel combo unit, Chattanooga, Guildford, Surrey, UK). The current was increased gradually until a nociceptive behavioral response was observed, or until the cut-off of $150 \mathrm{~mA}$ was reached. Sham-testing was performed by falsely turning the knob without increasing the current. Shamstimulation was stopped when a nociceptive response was detected by the observers or after 2 minutes.

b. TNT - a thermal probe (NTE-2A, Physitemp Instruments Inc., Clifton NJ, USA) was applied perpendicular to the surface of the skin at a temperature of $25^{\circ} \mathrm{C}$. The temperature was then decreased to $0^{\circ} \mathrm{C}$ so that the probe temperature gradually decreased to $0^{\circ} \mathrm{C}$. The temperature at which the dog responded to cold stimulation was recorded. If no response was observed, the test was stopped when the probe temperature reached $0^{\circ} \mathrm{C}$ to avoid tissue damage. Sham-testing was performed by disconnecting the probe from the controller so that it remained at room temperature.

c. MNT testing - MNT was tested using a commercial algometer (Bioseb, Vitrolles, France). An increasing pressure was applied with a flat tip (diameter of 3.5 $\mathrm{mm}$ ) perpendicular to the surface of the skin until a response was observed (MNT) or a cut-off pressure of 20 Newtons (N) N reached. This device did not allow sham-testing.

d. Assessment of DNIC - the endogenous pain inhibitory pathway was tested by comparing MNT (test stimulus) before (recorded during MNT testing as above) and after the application of a conditioning stimulus. Similarly to a technique previously described in human medicine, ${ }^{13}$ a neonatal blood pressure cuff was placed over the thoracic limb to be tested and inflated up to $200 \mathrm{mmHg}$ for $60 \mathrm{sec}$ onds. The pressure was released and MNT was recorded 3 minutes later. This test was not randomly allocated and was performed last in all dogs to avoid interference with other nociceptive testing. The order of the thoracic limb tested (left or right) was randomized and one limb was tested in the first period whereas the contralateral limb was tested in the second period.

\section{Feasibility}

Each QST measurement was evaluated for feasibility using a scale from 0 to 2 as similarly described. ${ }^{7}$ A score of 0 was given when there was a lack of cooperation or when the observer lack confidence in the data collected. A score of 2 corresponded to a combination of a clear response to the stimulus and strong confidence in the data collected. The score of 1 was given when the response was fairly clear or, when the observer was not entirely sure that the response corresponded to the stimulus.

\section{Statistical analysis}

Data analyses were performed using SAS (version 9.3; SAS Institute, Cary, NC, USA). Feasibility was expressed as a percentage corresponding to the number of measurements with scores 1 and 2, divided by the total number of measurements (excluding sham-testing) for each nociceptive testing. Nociceptive thresholds were expressed as mean \pm SD (scores 1 or 2 for both limbs and observers). Cut-off values were included in the statistical analysis when nociceptive behavioral responses were not observed during stimulation. The scores 1 and 2 for feasibility and reliability were analyzed together while scores 0 were excluded from further analysis. Intra- and inter-observer reliability and replicate repeatability were calculated using an intra-class correlation coefficient. Replicate repeatability was calculated using data from the first period of measurements. These results were expressed as ranges. For inter-observer reliability, results of all limbs for both periods (first and second set of measurements; 5 hours apart) were pooled together for each observer. A linear model for repeated measures with limb as a within-subject factor was used to test the effect of limbs (thoracic vs pelvic) on ENT and MNT; results for both observers and periods (first and second set of measurements; 5 hours apart) were pooled together. A linear model for repeated measures with observer, time (before vs after conditioning stimulus) and site of stimulation (left thoracic limb vs right thoracic limb) as within-subject factors was used to analyze the effect of the conditioning stimulus on MNT. This model was repeated using scores 2, as only one measure was taken for this test after the conditioning stimulus. For all comparisons, $P<0.05$ was considered to be statistically significant.

\section{Results}

\section{Animals}

Sixteen healthy client-owned dogs (six males and ten females) were included. Mean $\pm \mathrm{SD}$ (range) for age and 
body weight was $4.8 \pm 2.1$ years (2-9 years) and $32 \pm 16.7 \mathrm{~kg}$ (5.5-65.5 kg), respectively. The cumulative score for the severity domain and the interference domain of the CBPI was 0 for all dogs. The part "overall impression" of the CBPI ranged from excellent $(n=14)$ to very $\operatorname{good}(n=2)$. The scores for CPMS-SF ranged from 0 to 2.

\section{Feasibility}

Minimal restraint was required to perform QST. In one case, the second set of measurements for MNT could not be performed because the dog became restless. Feasibility was 99\% for ENT, $93.5 \%$ for MNT and $93.6 \%$ for TNT.

\section{QST}

Mean \pm SD for all limbs, observers and periods was 48.0 \pm 22.6 $\mathrm{mA}$ for ENT; $11.9 \pm 3.5 \mathrm{~N}$ for $\mathrm{MNT}$ and $8.5^{\circ} \mathrm{C} \pm 2.2^{\circ} \mathrm{C}$ for TNT (with exclusion of scores 0 and those cases where cut-off was reached for TNT without a behavioral response). Data for both observers and periods are presented in Table 1. Data could not be analyzed for TNT because results were inconsistent among dogs and many dogs did not respond to cold stimulation. MNT was significantly higher for the thoracic when compared with the pelvic limb $(P=0.002)$ (thoracic limb: 12.6 \pm 3.6 Newtons $(\mathrm{N})$; pelvic limb: $11.3 \pm 3.6$ $\mathrm{N}$ ), but there was no effect on site of stimulation for ENT. Sham-testing showed that $15 \%$ and $35.7 \%$ of ENT and TNT, respectively, were false-positive responses.

The conditioning stimulus was not significant $(P=0.15)$ when first assessed using both scores 1 and 2 . Values were significantly higher when the left thoracic limb was tested when compared with the contralateral limb (left ${ }_{\text {before }}: 13.3 \pm 4.1 \mathrm{~N}$; left $_{\text {after }}: 14.6 \pm 4.3 \mathrm{~N}$; right before $_{12.1 \pm 4 \mathrm{~N} \text {; right }}$ after $12.5 \pm 4.8$ N) $(P=0.04)$, but not between the two observers $(P=0.14)$. A significant difference was recorded when data were analyzed using scores 2 only (before stimulus: $12 \pm 4.7 \mathrm{~N}$; after stimulus: $14.3 \pm 4.2 \mathrm{~N})(P=0.049)$.

\section{Intra-observer reliability}

Intra-observer reliability for each limb ranged from $20.4 \%$ $70.4 \%$ for $\mathrm{Obs}_{1}$ and from $14.1 \%-56.4 \%$ for $\mathrm{Obs}_{2}$ using ENT, and $0 \%-71.5 \%$ for $\mathrm{Obs}_{1}$ and $39.9 \%-82.9 \%$ for $\mathrm{Obs}_{2}$ using MNT.

\section{Replicate repeatability}

The replicate repeatability for each limb ranged from $56.1 \%-93.7 \%$ for $\mathrm{Obs}_{1}$ and from $29.6 \%-86.8 \%$ for $\mathrm{Obs}_{2}$ using ENT, and $26.8 \%-70.4 \%$ for $\mathrm{Obs}_{1}$ and $48.8 \%-88.4 \%$ for $\mathrm{Obs}_{2}$ using MNT.

\section{Inter-observer reliability}

Inter-observer reliability was $91.4 \%$ for ENT and $60.9 \%$ for MNT.

\section{Discussion}

This study provides new insights on QST in healthy dogs. Information on ENT, MNT, TNT and the ability of a conditioning stimulus to assess the endogenous DNIC is reported as a new modality of QST in this species. The percentage of false-positives via sham-testing was also shown for ENT and TNT in addition to feasibility and reliability, and the replicate repeatability of each modality. Unlike previous studies, a score was attributed to each single measurement and feasibility was excellent ${ }^{14}$ for all modalities, which confirm that QST is practical and attainable even in untrained client-owned dogs.

The application of a conditioning stimulus increased MNT when only scores 2 were considered for analysis. Similar observations have been reported in monkeys, ${ }^{15}$ rats $^{16,17}$ and cats. ${ }^{18}$ The assessment of DNIC is a relatively new QST modality in veterinary medicine. ${ }^{19}$ Briefly, the DNICs are evoked by a painful stimulus and are responsible for the immediate and transient increase of nociceptive thresholds. ${ }^{16,20}$ The conditioning stimulus is commonly

Table I Mean \pm SD for electrical and mechanical nociceptive thresholds for thoracic and pelvic limbs using data from observers and period

\begin{tabular}{lllllll}
\hline $\begin{array}{l}\text { Nociceptive } \\
\text { stimulus }\end{array}$ & & First period & & \multicolumn{2}{l}{ Second period } \\
\cline { 3 - 4 } & & Thoracic limb & Pelvic limb & & Thoracic limb & Pelvic limb \\
\hline ENT (mA) & $\mathrm{Obs}_{1}$ & $51.1 \pm 30.2$ & $47.6 \pm 30.8$ & & $43.3 \pm 21.1$ & $44.8 \pm 20.9$ \\
& $\mathrm{Obs}_{2}$ & $55.4 \pm 30.5$ & $43.1 \pm 24.4$ & & $49.0 \pm 23.8$ & $46.0 \pm 27.2$ \\
MNT (N) & $\mathrm{Obs}_{1}$ & $12.0 \pm 4.7$ & $10.3 \pm 3.6$ & $10.2 \pm 3.2$ & $10.3 \pm 4.6$ \\
& $\mathrm{Obs}_{2}$ & $14.6 \pm 3.8$ & $12.4 \pm 5$ & $12.9 \pm 4.7$ & $11.9 \pm 4.2$ \\
\hline
\end{tabular}

Notes: First period, morning; second period, afternoon.

Abbreviations: ENT, electrical nociceptive threshold; mA, milliamps; MNT, mechanical nociceptive threshold; N, newton; Obs, observer I; Obs ${ }_{2}$, observer 2. 
applied in a remote location from the testing site and produces inhibitory responses at spinal and supraspinal levels. Therefore, changes in thresholds (ie, increased MNT in this study) are normally observed using a remote body location where stimulus is not applied (ie, contralateral limb). On the other hand, an ipsilateral conditioning stimulus (applied at a suprasegmental location in relation to where the test stimulus was conducted) was preferred in this study so patients would not have to be moved from one decubitus to the other. A previous study showed that the DNIC can be activated by the application of a conditioning stimulus both from a remote location on the contralateral or ipsilateral side, ${ }^{21}$ therefore our results are valid independent of the testing site.

Current recommendations on conditioned pain modulation testing state that the test stimulus should be performed shortly after the conditioning stimulus. ${ }^{22}$ MNT was measured 3 minutes after the conditioning stimulus to allow restoration of blood flow and normalization of skin temperature. It was judged to be sufficient to assess the DNIC and consequent changes in MNT. This study showed that the use of a conditioning stimulus and MNT are valuable in the assessment of DNIC as a novel QST modality in veterinary medicine. Future studies should be designed to identify the role of DNIC testing in dogs with osteoarthritis and neuropathic pain and in the study of underlying pain mechanisms and therapeutics.

Cold sensory testing (TNT) has been reported to have a low rate of responders following a stimulus at a fixed temperature of $0^{\circ} \mathrm{C}^{7}$ This study showed that using a ramped thermal nociceptive stimulus increased this response rate of healthy dogs. For comparison, the response rate obtained in the present study (all scores included) was $25.6 \%$ and $21.1 \%$ in the first and second period respectively, whereas the aforementioned study reported a response rate of $13.2 \%$ at the level of the plantar metatarsal region. ${ }^{7}$ This difference could also be explained by the fact that the testing sites were clipped in the present study, whereas authors applied the probe on unclipped areas in the previous study. ${ }^{7}$ Nevertheless, the response rate is overall low with a high percentage of falsepositive responses for TNT $(35.7 \%)$. The effect of observer, testing site or reliability could not be tested for TNT due to missing data and inconsistent response among subjects. Both observers provided lower scores during measurement which could show that behavioral changes associated with cold sensory testing are subtle and might require a complex learning curve. Another challenge in TNT testing was related to the device used. Decreases in probe temperature were faster than usually described in cold nociceptive testing in humans. ${ }^{23}$ Slower changes in temperature ramping $\left(1^{\circ} \mathrm{C} /\right.$ second $)$ could have improved the response rate and provided more robust results for this modality since the rate of temperature changes affects sensory thresholds in humans. ${ }^{24}$

TENS has been used in conscious ${ }^{25,26}$ healthy dogs. However, the modality is not commonly used in QST, which makes data comparison with other studies difficult. Different waveforms were applied in previous studies even when using similar testing sites and current frequencies. ${ }^{26}$ The VMS $^{\text {TM }}$ mode was used in this study and is characterized by short pulse duration that is designed to be more comfortable to the patient and suitable for application of high intensities. A gradual increase in current every 2 seconds was safe and well tolerated with excellent inter-observer reliability. Feasibility was excellent and superior to MNT and TNT. Additionally, stimulation requires minimal restraint and stress to the patient, and consistent behavior responses can be observed from a distance. Sham-testing revealed low false-positive responses associated with this modality. However, replicate effect was moderate and intra-observer repeatability was poor. This could suggest that individual variability can be commonly observed with TENS for ENT testing.

MNT values were consistent with findings using similar protocols and device. ${ }^{7,27}$ It has been shown that body weight influences MNT which could explain why higher nociceptive thresholds were recorded with this modality when compared with a previous study. ${ }^{7}$ However, feasibility was greater than previously reported, ${ }^{7}$ which could be related to the observers' ability to interpret behavioral changes associated with stimulation. Overall the technique was easy to perform and well-tolerated with moderate inter-observer reliability, which is consistent with other studies. ${ }^{6,7,27,28}$ Additionally, MNT was significantly higher on the first set of measurements when compared to subsequent periods in a different study. ${ }^{6}$ The present study does not corroborate this finding. However, intra-observer repeatability and replicate effect for MNT was observer-dependent and one could simply hypothesize that $\mathrm{Obs}_{2}$ was more consistent with measurements than $\mathrm{Obs}_{1}$ in this study. A statistical difference was found between thoracic and pelvic limbs thresholds but the magnitude of the difference was small. It was most likely due to technique-related variability and further studies are required to evaluate the possible clinical relevance of this finding.

\section{Conclusion}

This study provides additional information on QST in dogs using ENT, MNT and TNT. The use of a conditioning stimulus to assess the endogenous DNIC has also been reported with interesting results for future reference. Sham-testing allowed the assessment of false-positive responses with ENT and TNT. The study of QST using similar settings, modalities 
and laboratory conditions in dogs with naturally-occurring disease is warranted. It could provide valuable insight on the study of underlying mechanisms of disease and therapeutics.

\section{Acknowledgments}

This study was supported by Boehringer Ingelheim (Canada) Ltd and Mitacs through the Mitacs Accelerate Program, and the American Kennel Club Canine Health Foundation (grant ID 02353-A. An abstract of this paper was presented at the Association of Veterinary Anesthetists Spring Meeting in April 2017 as an oral presentation (Manchester, UK).

\section{Disclosure}

Hélène LM Ruel is the recipient of the Mitacs Accelerate Program which is partly sponsored by Boehringer Ingelheim. The authors report no other conflicts of interest in this work.

\section{References}

1. Uemura E. Fundamentals of Canine Neuroanatomy and Neurophysiology. 1st ed. Ames: John Wiley \& Sons Incorporated; 2015.

2. Yarnitsky D. Quantitative Sensory Testing. Muscle Nerve. 2004;46(4): 223-228.

3. Yarnitsky D, Granot M, Granovsky Y. Pain modulation profile and pain therapy: between pro- and antinociception. Pain. 2014;155(4):663-665.

4. Knazovicky D, Helgeson ES, Case B, Gruen ME, Maixner W, Lascelles BDX. Widespread somatosensory sensitivity in naturally occurring canine model of osteoarthritis. Pain. 2016;157(6):1325-1332.

5. Williams MD, Kirkpatrick AE, Griffith E, Benito J, Hash J, Lascelles BD. Feasibility and repeatability of thermal quantitative sensory testing in normal dogs and dogs with hind limb osteoarthritis-associated pain. Vet J. 2014;199(1):63-67.

6. Gorney AM, Blau SR, Dohse CS, et al. Mechanical and Thermal Sensory Testing in Normal Chondrodystrophoid Dogs and Dogs with Spinal Cord Injury caused by Thoracolumbar Intervertebral Disc Herniations. J Vet Intern Med. 2016;30(2):627-635.

7. Briley JD, Williams MD, Freire M, Griffith EH, Lascelles BD. Feasibility and repeatability of cold and mechanical quantitative sensory testing in normal dogs. Vet J. 2014;199(2):245-250.

8. Moore SA. Managing Neuropathic Pain in Dogs. Front Vet Sci. 2016;3(3):12.

9. Knazovicky D, Helgeson ES, Case B, et al. Replicate effects and testretest reliability of quantitative sensory threshold testing in dogs with and without chronic pain. Vet Anaesth Analg. 2017;44(3):615-624.

10. Brown DC, Boston RC, Coyne JC, Farrar JT. Development and psychometric testing of an instrument designed to measure chronic pain in dogs with osteoarthritis. Am J Vet Res. 2007;68(6):631-637.

Journal of Pain Research

Publish your work in this journal

The Journal of Pain Research is an international, peer reviewed, open access, online journal that welcomes laboratory and clinical findings in the fields of pain research and the prevention and management of pain. Original research, reviews, symposium reports, hypothesis formation and commentaries are all considered for publication.
11. Reid J, Nolan AM, Hughes JML, Lascelles D, Pawson P, Scott EM. Development of the short-form Glasgow Composite Measure Pain Scale (CMPS-SF) and derivation of an analgesic intervention score. Anim Welf. 2007 (16(Suppl):):S97-S104.

12. Bailey CS, Kitchell RL. Cutaneous sensory testing in the dog. J Vet Intern Med. 1987;1(3):128-135.

13. Graven-Nielsen T, Izumi M, Petersen KK, Arendt-Nielsen L. Userindependent assessment of conditioning pain modulation by cuff pressure algometry. Eur J Pain. 2017;21(3):552-561.

14. Portney L, Watkins M. Foundations of Clinical Research: Applications to Practice. 2nd ed. New Jersey: Prentice Hall Health; 2000.

15. Price DD, Dubner R, Hu JW. Trigeminothalamic neurons in nucleus caudalis responsive to tactile, thermal, and nociceptive stimulation of monkey's face. J Neurophysiol. 1976;39(5):936-953.

16. Le Bars D, Dickenson AH, Besson JM. Diffuse noxious inhibitory controls (DNIC). I. Effects on dorsal horn convergent neurones in the rat. Pain. 1979;6(3):283-304.

17. Dickenson AH, Rivot JP, Chaouch A, Besson JM, Le Bars D. Diffuse noxious inhibitory controls (DNIC) in the rat with or without pCPA pretreatment. Brain Res. 1981;216(2):313-321.

18. Morton CR, du HJ, Xiao HM, Maisch B, Zimmermann M. Inhibition of nociceptive responses of lumbar dorsal horn neurones by remote noxious afferent stimulation in the cat. Pain. 1988;34(1):75-83.

19. Monteiro B, Moreau M, Otis, L.P. DL, Pelletier JP, Troncy E. Quantitative sensory testing in animal models of chronic pain: A pilot study. Poster presented at: 6th International Congress of Neuropathic Pain. Sweden: Gothenburg; 2017.

20. Bannister K, Dickenson AH. The plasticity of descending controls in pain: translational probing. J Physiol. 2017;595(13):4159-4166.

21. Pud D, Sprecher E, Yarnitsky D. Homotopic and heterotopic effects of endogenous analgesia in healthy volunteers. Neurosci Lett. 2005;380(3):209-213.

22. Yarnitsky D, Bouhassira D, Drewes AM, et al. Recommendations on practice of conditioned pain modulation (CPM) testing. Eur J Pain. 2015;19(6):805-806.

23. Rolke R, Baron R, Maier C, et al. Quantitative sensory testing in the German Research Network on Neuropathic Pain (DFNS): standardized protocol and reference values. Pain. 2006;123(3):231-243.

24. Pertovaara A, Kojo I. Influence of the rate of temperature change on thermal thresholds in man. Exp Neurol. 1985;87(3):439-445.

25. Bergadano A, Andersen OK, Arendt-Nielsen L, Spadavecchia C. Noninvasive assessment of the facilitation of the nociceptive withdrawal reflex by repeated electrical stimulations in conscious dogs. Am J Vet Res. 2007;68(8):899-907.

26. Watabiki T, Nagakura Y, Wegner K, et al. Assessment of canine sensory function by using sine-wave electrical stimuli paradigm. Physiol Behav. 2010;101(3):327-330.

27. Knazovicky D, Helgeson ES, Case B, Gruen ME, Maixner W, Lascelles BD. Widespread somatosensory sensitivity in naturally occurring canine model of osteoarthritis. Pain. 2016;157(6):1325-1332.

28. Harris LK, Murrell JC, van Klink EG, Whay HR. Influence of experimental protocol on response rate and repeatability of mechanical threshold testing in dogs. Vet J. 2015;204(1):82-87.

\section{Dovepress}

The manuscript management system is completely online and includes a very quick and fair peer-review system, which is all easy to use. Visit http://www.dovepress.com/testimonials.php to read real quotes from published authors. 